\title{
Dengue Pathfinder: a real time web-based field dengue control e-surveillance system
}

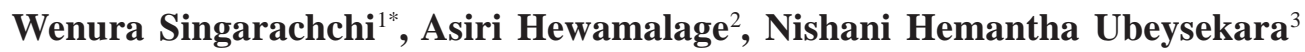 \\ ${ }^{1}$ Regional Director of Health Services Office, Galle District, Sri Lanka; ${ }^{2}$ Family Health Bureau, Ministry of \\ Health, Sri Lanka; ${ }^{3}$ Teaching Hospital Karapitiya, Sri Lanka
}

*Correspondence: wsingarachchi1967@gmail.com

(DDhttps://orcid.org/0000-0003-3161-9896

DOI: https://doi.org/10.4038/jccpsl.v26i2.8315

Received on 12 April 2020

Accepted on 04 June 2020

\section{Introduction}

Dengue is a mosquito-borne viral infection found in tropical and sub-tropical climates worldwide mostly in urban and semi-urban areas (1). The virus is transmitted to humans through the bites of infected female mosquitoes, primarily the Aedes aegypti and secondarily Aedes albopictus. Dengue prevention depends on effective vector control measures (2).

Dengue has become a global disease of public health concern and every year there are about 390 million dengue virus infections (3). In Sri Lanka, dengue is endemic in all 26 districts and had become one of the leading public health problems in the country (4). Dengue prevention strategies of the country include epidemiological surveillance for real time case detection, entomological surveillance for vector density forecasting and controlling the vector and integrated vector management (IVM) (5). Of these, case surveillance and entomological surveillance are the main pillars in relation to dengue.

\section{Case surveillance}

Dengue is a notifiable disease in Sri Lanka. Medical officer of health $(\mathrm{MOH})$ is the authorized officer for implementing dengue activities at grass-root level. In the routine notification system, information regarding a probable case is notified to the relevant $\mathrm{MOH}$ area from the hospital by the designated infection control nursing officer (ICNO). Once the notification form (H544) is received by the $\mathrm{MOH}$, they record it in the communicable disease notification register and hand over to the responsible public health inspector (PHI). The PHI does the field investigation and complete the field investigation form (H411) and hands it over to the supervising PHI (SPHI) together with the H544 form. After perusal, he submits the documents to the $\mathrm{MOH}$ to confirm the case. The confirmed case is recorded in the infectious disease register and compiled as the 'Weekly Epidemiological Return'(WER) (H399). Every Saturday, MOH sends the WER to the chief epidemiologist and the regional epidemiologist.

Sentinel surveillance sites are established in hospitals in high risk areas. Information of probable patients having fever and signs of haemorrhage is entered by the ICNO into an e-surveillance system 'Dengue Surveillance System (DenSys)'. The DenSys is a notification system which has been introduced to strengthen the existing surveillance. It aims to overcome issues in timeliness and accuracy. Further, special investigation form on dengue surveillance is filled by ICNOs to obtain more information on disease occurrence. 


\section{Entomological surveillance}

Entomological surveillance is carried out as routine surveillance, sentinel surveillance and spot checks. Selection of the method depends on the requirement of data for forecasting and controlling the disease. Inspection of the premises to identify probable breeding sites, larval surveys, pupal surveys, bioassays to check insecticide susceptibility, human landing catches, indoors and outdoors collection of adult mosquitoes to assess resting behaviour are carried out during surveillance. Special surveys are carried out six weeks prior and following the monsoon rains. The information gathered in pre-surveys in identified high risk gramaniladhari (GN) divisions is used to forecast and alarm public health teams implementing timely interventions to prevent the upcoming outbreak. The progress of interventions is monitored through post surveys.

\section{Gaps in the present dengue surveillance program}

With the escalation of dengue epidemics in the country, gaps in the paper-based surveillance system have been observed. This was mainly attributed to delays in notifications, delay in postal system and delay in documentation by health staff (ICNO, medical officers) due to increased workload. DenSys enables the receipt of patient information on the same day of the patient's admission and thereby initiates field investigations immediately. Similar to the paper-based system, it only gives the demographic data of the patient including the location of the focus up to the $\mathrm{MOH}$ division level. Still, the uploading of data into the system needs to be done manually while timeliness and accuracy of the data with manual entry to the system depends on the efficiency of hospital staff.

Although both these systems cater to the need of systematic collection of data, they do not address most of the prevailing issues of real-time field dengue control activities. Moreover, entomological surveillance system is still paper-based and there is delay in action due to reporting delays. To fill-up the gaps in current systems, a new software has been developed: "Dengue Pathfinder". It is a comprehensive real time e-surveillance system, from notification of disease to the control, monitoring, outbreak prediction and modelling with a real time epidemic tracking system.

\section{The Dengue Pathfinder}

The Dengue Pathfinder consists of a web application as well as a mobile application. The first component of the application is the notification phase, which allows 'ICNO', 'Laboratory', 'General Practitioners', 'Private Hospital', 'Range PHI' and '(patient) self' notification of suspected dengue cases through mobile notification to field level service providers. Once the case information is uploaded as a notification through web or mobile application, the data will be stored in a secured database. This generates a mobile notification to all the PHIs in a given $\mathrm{MOH}$ area through the mobile application as an "application notification". When the correct range PHI accepts the application notification, after scrutinizing the address, the system ignores the others. Automatically the notification register is generated as an accepted list and the geographical information of the case is depicted in a spot map once the PHI accepts and gets the global position system (GPS) coordinate by visiting the place.

Second component of the application is the field investigation phase. PHI has already got the GPS coordinates and will complete the PHI field investigation e-form online while doing the field investigation. Then he should complete the action sheet and upload images of the primary focus of vector breeding. The PHIs are expected to complete this ideally within 24 hours of notification or at least by three days.

Third component of the application is the intervention phase. Once the PHI completes the field dengue control measures, he should complete the intervention axis components of the app and submit to $\mathrm{MOH}$. The $\mathrm{MOH}$ should submit an e-mail notice of action to the relevant responsible institution. The 'standard email templates' and list of institutions are in-built to the master data with relevant e-mails. The institution can be selected by the $\mathrm{MOH}$ by going through a drop-down list of institutions. All the institutions registered in master data have been given training on Dengue Pathfinder. Trained person in the institution has been assigned to control dengue in the institution and report the progress through the app, which will automatically appear in the $\mathrm{MOH}$ consolidated data.

The most fascinating fourth component in this application is the monitoring phase where $\mathrm{MOH}$ can 
monitor the whole process from his office. He can visualize the consolidated data, supervise each and every case investigation and produce activity maps.

In the analysis phase of the application, field investigations of PHIs are automatically audited. This can be monitored by the $\mathrm{MOH}$ on his 'dashboard' and are reproduced as charts, tables and location maps. Further, it will generate reports on the source of infection, percentage positivity of 'within premises', 'immediately outside', 'common source', 'outside the GN area', 'outside the MOH' and 'outside the district'. These are very important program indicators in field dengue control which are underutilized by the current system. Moreover, 'the indigenous incidence', 'indigenous migrant infectivity rate' and 'migrant infectivity rate'. Others are 'automatically generated and available as monthly bar-charts'. There are options to filter the reports based on time and geographical location. The regional director, consultant community physician and the regional epidemiologist will do the audit on field control measures taken by the $\mathrm{MOH}$ and the overall system monitoring will be done online on their dash boards. Apart from the case surveillance, the Dengue Pathfinder provides opportunity to consolidate entomological surveillance. Thus, the sixth component of the app is the entomological surveillance. The health entomological officers (HEO) have already been given the list of cases as app notification pertaining to their $\mathrm{MOH}$ area. Hence, the HEO knows the reported location addresses of all dengue cases in his area. This is helpful to plan the case-based spot surveys in households. The HEO has given the access to the electronic field entomological survey form online where he can enter the survey data and images. This information is visible to the entomologist in his/her dashboard as 'consolidated ento-survey data'. After finishing dissection of the mosquitos and larvae, the species can be entered by editing 'ento-survey data' by the HEO or entomologist. Once this is completed, the Dengue Pathfinder reports all available entomological indices including container positivity which can be filtered based on time and geographical location. In addition, the geographic information system (GIS) maps are generated automatically. All these are location spot maps which can be used as important instruments to identify the spatial propagation of the epidemic or outbreaks with time. Further, it measures the effectiveness of active field interventions while the outbreak is mitigated at field level.

\section{Public health implications of the dengue pathfinder}

The Dengue Pathfinder provides a platform to network between pertinent stakeholders such as the Ministry of Health, regional public health staff, hospitals, local governments and law enforcement authorities in controlling dengue. This will strengthen inter-sectorial collaboration. The real-time data will help the field officers in outbreak forecasting, immediate action, and timely mitigation. Retrieval of data in this type of a database will help to predict outbreaks and mitigate them timely. This will save capital cost and address the focal field activity with limited human resource.

This e-surveillance system alleviates many shortages of the existing dengue surveillance systems of the country. It minimizes data entry errors via validation, enables easy follow up and track incidence, take management decisions on reports, audit PHI field performance, online field activity, role-based access control for reporting, data security and minimize the cost of field activity on prevention.

This had been pre-tested in a few $\mathrm{MOH}$ areas of Galle District and is recommended to be disseminated after correcting the identified pragmatic issues in the modified version 'Dengue Pathfinder Version-3' with a 'window of public complaint pertaining to dengue and online 'dengue death surveillance system'. This was an iterative process overcoming constrains specially at institutional level and satisfying the main stakeholders on concurrence on field testing and application.

The Dengue Pathfinder combines all the steps of an effective surveillance system for any communicable disease including notification, field investigation, implementation of the intervention, monitoring, data analysis and evaluation, and vector surveillance. Hence, future escalation to all types of communicable disease surveillance can be done in a 'single application' on different platforms. It will facilitate high quality comprehensive real-time data for interventions.

\section{References}

1. World Health Organization. Comprehensive Guidelines for Prevention and Control of Dengue and Dengue Haemorrhagic Fever: Revised and Expanded Edition. SEARO Technical Publication Series. 2011: 60. 
2. World Health Organization. Dengue and severe dengue. Available from: https://www.who.int/news$\mathrm{room} /$ fact-sheets/detail/dengue-and-severedengue.

3. World Health Organization. Dengue Guidelines for Diagnosis, Treatment, Prevention and Control. New Edition. France: World Health Organization, 2009. Available from: https://www.who.int/tdr/ publications/documents/dengue-diagnosis.pdf.
4. Epidemiology Unit of Sri Lanka. Dengue update. Epidemiology Unit. 2020. Available from: http:// www.epid.gov.lk/web/index.php?option=com content\&view=article\&id=171\%3Adengue-update \&catid=51\%3Amessage-for-public\&Itemid= 487\&lang=en.

5. National Dengue Control Unit. National Action Plan on Prevention and Control of Dengue 2019 - 2023. Sri Lanka. Colombo: Ministry of Health, 2019. 